\title{
Simulation Studies of Entropy-Driven Crystallization in Athermal Chain Packings in the Bulk and under Confinement ${ }^{+}$
}

\author{
Miguel Herranz, Pablo Ramos, Katerina Foteinopoulou, Nikos Karayiannis and Manuel Laso \\ Institute for Optoelectronics and Microtechnology (ISOM) and ETSI Industriales, Universidad Politecnica de \\ Madrid (UPM), José Gutierrez Abascal 2, Madrid, Spain \\ † Presented at the Entropy 2021: The Scientific Tool of the 21st Century, 5-7 May 2021; Available online: \\ https://sciforum.net/conference/Entropy2021/.
}

Published: 5 May 2021

We present results from extensive off-lattice simulations on packings of flexible linear chains of hard spheres in the bulk and under confinement. We employ a Monte Carlo scheme, built around advanced, chain-connectivity-altering moves, for the short- and long-range equilibration even for very long and definitely entangled systems, at very high concentrations near the maximally random jammed (MRJ) state and under extreme confinement [1]. Local environment and similarity to specific crystal structures are gauged through the crystallographic element norm (CCE) metric [2] which is able to distinguish between different competing crystal structures. The established crystal morphologies range from random hexagonal close packed ones with a single or varied stacking direction(s) to pure facecentred cubic (fcc) and hexagonal close packed (hcp) crystals. We explain how the total entropy of the system increases as the local environment of the crystal phase becomes more symmetric and spherical. This entropic effect leads to the observed transition from the initial amorphous to the final crystal phase [3]. By extending the simulations to trillions of steps crystal perfection is observed in accordance to the Ostwald's rule of stages in crystal polymorphism.

In general, bond tangency of successive monomers along the chain backbone or the corresponding gaps affect profoundly the ability of chains to crystallize [4]. Based on these findings, by using simple geometric arguments, we explain the role of rigid and flexible constraints in the packing behavior (crystal nucleation and growth) of general atomic and particulate systems.

\section{References}

1. Ramos, P.M.; Karayiannis, N.C.; Laso, M. Off-lattice simulation algorithms for athermal chain molecules under extreme confinement. J. Comput. Phys. 2018, 375, 918-934.

2. Karayiannis, N.C.; Foteinopoulou, K.; Laso, M. The characteristic crystallographic element norm: A descriptor of local structure in atomistic and particulate systems. J. Chem. Phys. 2009, 130, 074704.

3. Karayiannis, N.C.; Foteinopoulou, K.; Laso, M. Entropy-driven crystallization in dense systems of athermal chain molecules. Phys. Rev. Lett. 2009, 103, 045703.

4. Karayiannis, N.C.; Foteinopoulou, K.; Laso, M. The role of bond tangency and bond gap in hard sphere crystallization of chains. Soft Matter 2015, 11, 1688-1700.

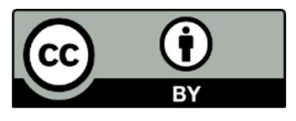

(C) 2021 by the authors. Licensee MDPI, Basel, Switzerland. This article is an open access article distributed under the terms and conditions of the Creative Commons Attribution (CC BY) license (http://creativecommons.org/licenses/by/4.0/). 